\title{
LA HISTORIA ECONÓMICA EN URUGUAY: DESARROLLO Y PERSPECTIVAS
}

\author{
LUIS BÉRTOLA \\ Universidad de la República-Uruguay
}

\section{RESUMEN}

Este artículo aborda el desarrollo reciente de la Historia Económica en Uruguay, particularmente los aspectos de conformación institucional y las líneas teórico-metodológicas y temáticas predominantes. Después de identificar una etapa fundacional de la disciplina entre 1960 y 1985 se realiza una evaluación del desarrollo reciente, al que se caracteriza como un período de dispersión, refundación y especialización. Se concluye que, a pesar de que la Historia Económica perdió su papel de nucleador de las ciencias sociales y de que el número relativo de sus cultores ha disminuido, la disciplina ha experimentado un proceso de consolidación institucional y organizativa. Éste abarca la formación especializada a nivel de posgrados, un estrecho relacionamiento con la comunidad internacional y el esfuerzo por combinar el conocimiento histórico con el rigor teórico y metodológico. Sobre estas bases se ha venido incrementando la producción y es de esperar que la disciplina vuelva a ganar un lugar importante en la vida académica.

\section{ABSTRACT}

This paper is concerned with recent development of Economic History in Uruguay. It focuses on its institutional features as well as on the prevailing theoretical and methodological approaches and research topics. A foundational period 1960-1985 is shortly reviewed. Recent development is thereafter discussed and characterised as one of dispersion, re-foundation and specialisation. The paper concludes that, notwithstanding Economic History loosed its central place and weight among the social sciences, the discipline has gone through a process of institutional and organisational strengthening. This embraces specialised graduate education, fluent relationships to the international community and an attempt to combine historical knowledge with rigo- 
rous treatment of theory and method. On this basis, the production of more and better facts has been increasing and, hopefully, the discipline may regain an outstanding place in academic life.

\section{INTRODUCCIÓN}

Este artículo aborda, de manera muy sintética, el desarrollo reciente de la Historia Económica en Uruguay, particularmente los aspectos de conformación institucional y las líneas teórico-metodológicas y temáticas predominantes. Por desarrollo reciente se entiende a lo sucedido desde mediados de los años ochenta, a partir de la redemocratización del país y de la restauración de la vida académica que ello trajo aparejado. En dos secciones previas se prepara el escenario. En la primera se presenta concisamente una definición de la Historia Económica, a los efectos de dejar en claro la referencia de nuestra reflexión. En la segunda se presenta una síntesis apretada de la historia anterior a lo que se ha llamado «reciente». A modo de conclusión se presenta una breve evaluación del estado actual de la disciplina y criterios para una agenda de desarrollo de la misma.

\section{La Historia Económica: una definición}

A riesgo de demorar la entrada en tema, pienso que una definición de la disciplina es fundamental para evaluar su recorrido y percibir sus perspectivas. El tema central, el objeto de la Historia Económica, es explicar lo que North llama «la forma desigual y errática que toma el cambio histórico y el desarrollo contemporáneo y el cómo modelizar, interpretar, regularizar ese proceso de cambio y desarrollo»" ${ }^{1}$.

La Historia Económica es la disciplina que encuentra su núcleo de interés en el desarrollo de los sistemas económicos y sus diferentes componentes: la producción, el intercambio, la distribución y el consumo, entendiendo a este último como un proceso mediante el cual, al decir de Sen (1998), se producen diferentes modos de vida en base a la utilización de diferentes medios de vida producidos. La Historia Económica pretende explicitar las leyes que rigen estos procesos, las etapas de su desarrollo, las determinantes de su surgimiento y decadencia, su especificidad histórica

1 North (1994), p. 1. 
y, por qué no, las determinantes universales de la reproducción económica y social, las leyes económicas más generales.

Existe un continuo de situaciones temporales que van desde el muy largo plazo hasta las coyunturas de muy corto plazo y desde el pasado remoto hasta el hecho reciente, que demandan un conocimiento económico-social complejo. Se trata del fruto de un proceso deductivo e inductivo en el que los diferentes determinantes del proceso de cambio económico son tenidos en cuenta: las relaciones sociales y su relación con los recursos naturales, los arreglos institucionales, las racionalidades limitadas de los agentes, las determinantes del cambio tecnológico, por mencionar solamente algunos elementos claves de manera sintética y no articulada. Estos elementos son parte del análisis, independientemente de la dimensión temporal del estudio, de la época en estudio y de la ubicación geográfica. En este sentido, la diferencia entre Economía e Historia Económica parece artificial, y la jerarquía más clara en la diferenciación es aquella que surge de las diferentes instancias de la investigación, del proceso de producción de conocimientos: la modelización, el testeo de hipótesis y empírico, la narración del hecho histórico concreto, que devuelve y realimenta el proceso de generalización y modelización, de elaboración de evidencia, de hipótesis, de recomendaciones u opiniones acerca de las decisiones siempre contingentes de los actores individuales y colectivos.

El historiador económico ha de conocer, dominar y desarrollar la teoría económica universal y se habrá de especializar en aspectos de la teoría económica y en épocas específicas, con sus propias demandas teóricas y metodológicas. En este contexto se puede coincidir con la afirmación de McCloskey (1996) -la que, sin embargo, no puede dejar de verse más como una aspiración que como una realidad - sobre que la Historia Económica sirve para producir más evidencias de hechos económicos, mejores evidencias de hechos económicos, mejor teoría económica, mejor política económica y mejores economistas. Igualmente puede rescatarse el razonamiento espejo de Crafts (1999), acerca de que la ciencia económica aporta a la Historia Económica más y mejores hechos económicos, mejores hipótesis, mejores interpretaciones históricas y, finalmente, mejores historiadores.

\section{Una breve historia de la Historia Económica en el Uruguay}

Sin duda, intentar realizar una periodización del desarrollo de la Historia Económica en Uruguay (o en cualquier parte) conlleva un conjunto 
de dificultades. La reflexión sobre las características teórico-metodológicas de la disciplina en Uruguay tiene muy pocos años y no existen muchos antecedentes de trabajos que reflexionen al respecto ${ }^{2}$. En esta sección haremos una breve referencia al período que llamamos prefundacional y nos concentraremos en el que llamamos integrador y fundacional, desde 1960 hasta la salida de la dictadura militar.

Llamamos prefundacional al período anterior a 1960 en el sentido de que la Historia Económica no se reconocía como un campo específico de investigación o de docencia y en el sentido de que las contribuciones al conocimiento histórico económico se producían de manera claramente subordinada al desarrollo de otras disciplinas del conocimiento. En el trabajo ya referido Camou y Moraes aluden de manera muy bien documentada a las contribuciones de los economistas y los historiadores en este período. Igualmente se mencionan contribuciones de intelectuales y políticos no fácilmente ubicables disciplinariamente ${ }^{3}$.

Los economistas, predominantemente dedicados al estudio de aspectos monetarios y fiscales, no acompañaban su investigación con reflexiones referentes a los aspectos del cambio histórico, sino que parecían orientar la investigación a recomponer una base informativa básica acorde a los requerimientos teóricos y metodológicos que imponía la reciente instauración en el medio de la ciencia económica como profesión. En esta dirección hicieron muchas veces recorridos históricos largos en los que, sin pretender sobreinterpretar un silencio reflexivo, podríamos decir que predominó lo que Arrow llamó una etapa naif de la visión de la Historia Económica por parte de los economistas, es decir, verla como un campo de pruebas de la teoría ${ }^{4}$.

Por parte de los historiadores, si bien se venía gestando el surgimiento de la Nueva Historia con la prefesionalización del historiador, los pocos trabajos sobre historia económica no pretendían aún constituir a los fenómenos económicos en una clave interpretativa del acontecer histórico, ni manejaban herramientas teóricas capaces de ello, como lo harían posteriormente en el período fundacional e integrador.

Si bien la dictadura militar impuso condiciones particulares a la investigación y, de no mediar la misma, el desarrollo probablemente hubiese seguido derroteros muy distintos, pensamos que en términos generales lo producido durante el período militar mantuvo una muy fuerte continuidad

\footnotetext{
${ }^{2}$ Por una reciente contribución ver M. M. Camou y M. I. Moraes (1998).

"Camou llama a este período «Los pasos iniciales».

4 Arrow (1985), p. 323.
} 
con los paradigmas vigentes en los años sesenta. Por ello, aunque matizando a su interior, optamos por considerar 1960-1985 como una período relativamente homogéneo en el que aún no predominaban los rasgos del período posterior ${ }^{5}$. Puede sostenerse que los años sesenta constituyeron un período de muy fuerte desarrollo de la Historia Económica en Uruguay, un verdadero período fundacional de la disciplina. Ello no implica despreciar diversas contribuciones realizadas por autores muy variados en períodos anteriores.

Este período fundacional resultó de la confluencia de un conjunto muy variado de factores. Desde el lado de los historiadores, predominó una combinación de diferentes tradiciones historiográficas y teóricas que hacían del elemento económico y social el articulador de todo pensamiento histórico. La fuerte influencia del pensamiento de los Annales, en armonía con la tradicional influencia cultural francesa en Uruguay, había llevado a la preocupación por la incorporación de las ciencias sociales a la interpretación histórica y a la ruptura con la historiografía política pragmática. Por otra parte, la crisis del modelo industrializador y las limitaciones para mantener el crecimiento económico y las expectativas de profundizar las políticas de bienestar social enfatizaron la importancia de la problemática económica, a la vez que jerarquizaron la búsqueda de las raíces de la situación crítica que se estaba viviendo. Estas raíces se rastreaban hasta la herencia colonial y las particulares modalidades de la inserción uruguaya al mercado mundial a fines del siglo XIX. Sin subestimar otras contribuciones, permítasenos sostener que los más elocuentes exponentes de las contribuciones de los historiadores en este período son dos conjuntos de obras elaboradas por dos equipos de investigadores. Por un lado, los rigurosos trabajos de clara inspiración marxista de De la Torre, Rodríguez y Sala de Tourón, concentrados en la historia colonial y el período de la revolución independentista, particularmente en el estudio de las elites económico-sociales y en la estructura de la propiedad de la tierra ${ }^{6}$. Por otro lado, la colección de siete volúmenes de Barrán y Nahum (1967-1978) sobre el período 1850-1913, un trabajo más ecléctico e igualmente riguroso. Si bien esta colección fue culminada fuera del período que estamos analizando y presenta algunos cambios de conceptualización en su transcurso, mantiene las líneas interpretativas y metodológicas generales a lo largo de toda la

\footnotetext{
' Camou y Moraes optan por una división del período en pre y post 1973.

- De la Torre, Rodríguez y Sala de Tourón (1971); Sala de Tourón, De la Torre y Rodríguez (1967); Sala de Tourón, Rodríguez, De la Torre (1967); De la Torre, Sala de Tourón y Rodríguez (1972); Sala de Tourón, De la Torre, Rodríguez (1978).
} 
obra. Vale la pena detenerse en este trabajo por el difícilmente sobreestimable impacto que tuvo, tiene y por mucho tiempo mantendrá en la historiografía y sentido histórico nacional uruguayos. Se conjugan en él aspectos tales como la mentalidad de los estancieros, la estructura de la propiedad de la tierra, el peso de la clase mercantil montevideana y la dependencia económica (comercial, financiera, etc.) en una rica e ilustrativa explicación del desempeño económico-social del período.

Entre los economistas de los años sesenta predominaban diferentes tipos de interpretaciones marxistas, estructuralistas, desarrollistas y dependentistas. Todas ellas eran corrientes que ponían fuerte énfasis en los enfoques longitudinales; todas ellas recurrían a diferentes formas de determinación histórica para explicar la situación en que se encontraba América Latina; todas ellas demandaban del conocimiento histórico como parte de su construcción teórica. La profesión de economista, aún con pocos años de implantación institucional, se vio fuertemente atraída hacia enfoques teóricos de corte keynesiano, desarrollista y luego dependentista. Los enfoques de los economistas eran gustosamente de largo plazo. Los libros se titulaban «desarrollos», «procesos», «evoluciones» de los objetos en cuestión, e insistentemente buscaban en la herencia colonial y en las formas de inserción a la economía internacional a fines del siglo pasado el origen de las estructuras económicas del subdesarrollo, del desequilibrio productivo y de la dependencia. En particular, los trabajos de los economistas se concentraron en estudios de las determinantes del crecimiento económico y la industrialización desde los años treinta, pero muy frecuentemente se realizaban ensayos interpretativos del desarrollo anterior. Además del conjunto de trabajos y diagnósticos realizados por la muy cepalina CIDE (Comisión de Investigaciones y Desarrollo Económico) ${ }^{7}$, que iniciaron el proceso de construcción de las cuentas nacionales, pueden destacarse el estudio de Faroppa (1965) sobre las condicionantes del crecimiento industrial y el cambio estructural desde los años treinta y el muy influyente marxista y dependentista trabajo colectivo del Instituto de Economía, concentrado en el estudio de las determinantes del estancamiento agropecuario y del crecimiento industrial ${ }^{8}$. También desde otras ciencias sociales surgieron destacados aportes a la Historia Económica y Social del Uruguay, ya sea que tuvieran un enfoque más sociológico, politológico o geopolítico. También desde el campo de la acción política surgieron trabajos inter-

${ }^{7}$ Uruguay, CIDE (1963). Ver también sobre el sector agropecuario, Uruguay, MGA, OPYPA-CIDE (1967).

${ }^{8}$ Uruguay, Universidad de la República, Instituto de Economía (1969). 
pretativos de interés, en particular los del socialista Vivián Trías y el comunista Rodney Arismendi.

En síntesis, las ciencias sociales en un sentido amplio, se constituyeron en una herramienta de articulación del discurso histórico a la vez que la Historia se constituyó en un campo natural del desarrollo de las ciencias sociales. Como resultado de todo ello existió una verdadera convergencia hacia la Historia Económica y Social, tanto desde la Economía, la Sociología y la Ciencia Política como desde la Historia.

Una característica de la producción de este período que corresponde señalar fue su carácter predominantemente macroeconómico. Eventualmente fue sectorial, pero fuertemente vinculado a una visión macroeconómica y macrosocial. Pudo tratarse del desarrollo económico general, o de la cuestión agraria, o del desarrollo industrial: temas éstos centrales de la investigación, pero la perspectiva microeconómica estuvo ausente.

Otro elemento característico de este período fue que si bien coincidió con una especie de «latinoamericanización» de la conciencia de una sociedad que se había creído más bien europea, este proceso se centró en una búsqueda obsesiva de la particularidad uruguaya, probablemente asociada a los problemas de su débil identidad nacional. Así, a pesar del predominio de marcos teóricos generalizantes y propensos a integrar la sociedad uruguaya en una matriz histórica común a las latinoamericanas, la investigación se mantuvo con rasgos sumamente particularistas y muy poco comparativos.

La ruptura del orden democrático en 1973 y la intervención de la Universidad de la República por parte de la dictadura militar habrían de aparejar importantes cambios en la actividad académica: cierre de carreras, persecución y exilio masivo de investigadores, lenta creación de centros privados de investigación en ciencias sociales en los que se replegaron los proscritos de la universidad pública, creciente financiación externa de la actividad de investigación. Los centros privados habrían de generar, principalmente en los primeros años de la década de los ochenta, una significativa producción que no se deja asimilar fácilmente a las características de la investigación de los años sesenta y muchas veces ya encontramos en su producción los gérmenes de desarrollos posteriores.

No puede sostenerse, sin embargo, que en estos años se haya producido un cambio radical e inmediato de los temas y modalidades de investigación. Por el contrario, las principales obras siguieron estando estructuradas, aunque con tonos ideológicos menos estridentes, en torno a las mismas tradiciones y preguntas que en el período anterior. En todo caso, la investigación se tornó aún más rigurosa y precisa desde el punto de vista del 
manejo de fuentes históricas, aunque ésta es una afirmación un tanto audaz que pretende captar una tendencia general más que aplicarse a todos los casos por igual. Es posible que este desarrollo se pueda vincular al hecho de que los estudios macroeconómicos fueron progresivamente dando lugar y complementándose de manera creciente con estudios sectoriales y monográficos, tanto en los trabajos provenientes del área histórica como de las ciencias sociales. Igualmente, la investigación adquirió caracteres más desideologizados y varias investigaciones lograron presentar abundante evidencia empírica e interpretaciones que contradecían muchas verdades panfletarias acuñadas en el período anterior. A su vez, una connotación negativa fue un cierto empobrecimiento de las discusiones teóricas, parcialmente fruto del aislamiento intelectual con respecto a diversos debates desarrollados en las ciencias sociales latinoamericanas y a nivel mundial a fines de los setenta e inicios de los ochenta.

En estos años aparece y prácticamente se concluye la segunda serie de volúmenes de Barrán y Nahum, serie que abordara una temática más variada que la del Uruguay rural, aunque sin transgredir el período antes estudiado. En el primer volumen de esta colección se presenta una interesante tesis demográfica, aún poco discutida e interpelada, a la vez que se insinúa la incursión de Barrán en temas de mentalidades, sensibilidades y profesiones, con los que habria de revolucionar la producción historiográfica y el mercado editorial uruguayos en los años noventa. Igualmente, y también desde el campo de la Historia, se produce la incursión de Raúl Jacob con obras importantes, en base a un riguroso trabajo empírico que abrió nuevos campos de investigación y contribuyó a destruir algunas caracterizaciones excesivamente simplistas ${ }^{9}$.

En este período comenzó a producirse cierto abandono de la problemática histórica por parte de los economistas, quienes se vieron naturalmente atraídos por el estudio de los cambios producidos en la economía uruguaya en los años de dictadura militar. Sin embargo, ni el cambio de paradigma que habría de ser notorio posteriormente se insinuaba todavia con claridad ni los trabajos de largo plazo desaparecieron de la escena. Un ejemplo de esto último es el trabajo de Danilo Astori (1979) sobre la problemática tecnológica en la ganadería uruguaya, trabajo acompañado de similares esfuerzos realizados en centros privados, como CIEDUR y CINVE ${ }^{10}$. Otro intento destacable por relanzar el interés de los econo-

${ }^{9}$ Ver, por ejemplo, JACOB (1981) y (1983).

${ }^{10}$ CIEDUR (Centro Interdisciplinario de Estudios sobre el Desarrollo, Uruguay), CINVE (Centro de Investigaciones Económicas). 
mistas por la problemática de largo plazo, aunque éste sí ya expresando cambios de paradigma, es el volumen editado por la Academia Nacional de Economía (1984), reuniendo trabajos de diversos economistas. Un trabajo que combinó el interés por el largo plazo al tiempo que se ubicaba en el emergente paradigma neoclásico, fue un estudio sobre el proteccionismo desarrollado en el marco del Banco Central del Uruguay ${ }^{11}$.

Particularmente fértil fue, hacia el final del período dictatorial, la contribución a temas de historia económica y social desde otras ciencias sociales, tales como la demografía, la ciencia política, la sociología, cultivadas en un ambiente interdisciplinario en los distintos centros privados de investigación (CIESU y CLAEH ${ }^{12}$, además de los ya mencionados CIEDUR y CINVE), a menudo en un estrecho contacto con historiadores. Esa interacción alentó a los cientistas sociales a realizar importantes contribuciones al conocimiento histórico, a la vez que obligó a los historiadores a un diálogo más estrecho con las ciencias sociales.

Como fruto de este proceso fundacional e integrador, y como forma de sintetizar sus logros, quiero resaltar dos obras que resultaron paradigmáticas para el cultivo de la disciplina. El primero de ellos es el trabajo de Julio Millot, Carlos Silva y Lindor Silva (1973). Ese trabajo representa una síntesis de tres aspectos programáticos de la disciplina: el manejo de teoría económica y de preguntas claramente expuestas; el desarrollo de una metodología que combina la crítica de las fuentes con un sistemático esfuerzo de construcción de indicadores cuantitativos, y el fluido manejo de las circunstancias históricas, tanto económicas como políticas, sociales e institucionales. Los dos primeros elementos distinguen este trabajo de la gran mayoria de las contribuciones hechas por historiadores, en tanto el segundo y tercer elemento marcan importantes diferencias con los trabajos de los economistas del período, que si bien no faltos de contextualización histórica y de manejo cuantitativo, no realizaron en esos planos entradas fuertes y $\tan$ bien equilibradas. El otro trabajo tiene características peculiares, por ser su autor un inglés radicado en Inglaterra y que, por tanto, no constituye un producto propio de las ciencias sociales en Uruguay. Sin embargo, la obra de Henry Finch (1980) sin duda recoge de manera muy armónica los diferentes aportes a la Historia Económica realizados por las diferentes vertientes reseñadas anteriormente. Es un trabajo fiel a la idea de que no hay Historia Económica sin teoría económica y que

${ }^{11}$ Anichini, Caumont y Sjaastad (1977).

12 CIESU (Centro de Investigación y Estudios Sociales del Uruguay), CLAEH (Centro Latinoamericano de Economía Humana). 
recurre fluidamente al manejo de la teoría no exclusivamente económica. En ese sentido se inscribe en la tradición de la Economía Política, hecho claramente expuesto en el título de la versión inglesa. Es un trabajo que realiza muy importantes contribuciones en materia de construcción de series cuantitativas, particularmente en lo que se refiere al comercio exterior. Igualmente, es un trabajo que demuestra haber captado toda la complejidad y especificidad del entramado de relaciones sociales e institucionales en sus particulares contextos históricos de una manera que ha sorprendido a sus colegas uruguayos.

\section{El desarrollo reciente: dispersión, refundación y especialización}

He definido como desarrollo reciente a lo acontecido a partir de la reapertura democrática a mediados de los años ochenta. Sin duda es muy difícil establecer cortes claros en la actividad intelectual colectiva y toda periodización tiene algo de arbitrario. Así como encontramos en la producción de los tempranos años ochenta elementos de la década anterior, no dejamos de percibir cambios importantes que probablemente justifiquen una divisoria más clara en relación a los años sesenta. Igualmente, no podemos dudar de que una buena parte de lo que se habrá de considerar en este período tiene no solamente sus raíces y orígenes en momentos anteriores, sino también trabajos con los que tiene continuidad.

He caracterizado a este período como de dispersión. Con ello se hace referencia a la clara pérdida del papel nucleador de las ciencias sociales que había detentado la Historia Económica en los años sesenta. También he caracterizado a este período como de refundación, porque se ha producido un proceso de consolidación académica de la disciplina. Igualmente he caracterizado a este periodo como de especialización, porque de la síntesis de ambas caracterizaciones anteriores ha resultado una propuesta que, si bien no es aislacionista con respecto a las demás ciencias sociales e históricas, sí ha representado un proceso de profesionalización de la disciplina. Para abordar con más claridad estos tres aspectos analizaremos las líneas teórico-metodológicas y temáticas predominantes y los aspectos de conformación institucional en torno a la disciplina. Comencemos por esto último para dejar claro el mapa. 


\section{El entorno institucional}

Que la historia reciente no se deja presentar de manera simple lo confirma su historia institucional. No se habla aquí de lo institucional a la manera de North, sino en un sentido más laxo, que incluye a las organizaciones. También he de limitarme a realizar consideraciones que tienen exclusivamente en cuenta el impacto sobre la Historia Económica.

Sin duda la vuelta a la democracia tuvo un impacto muy importante en la vida académica. La recuperación de la autonomía de la principal y absolutamente dominante universidad pública se vio acompañada del retorno a su seno de los académicos activos en los centros privados y de los viejos académicos que habían debido exiliarse. También se incorporaron nuevas generaciones de académicos formados en el exterior. A su vez, se produjeron cambios de estructura de la mencionada universidad, cuya trascendencia sería significativa, en particular la creación de la nueva Facultad de Ciencias Sociales, coexistiendo con la de Humanidades y Ciencias de la Educación y la de Ciencias Económicas y Administración.

Sin embargo, el proceso de recomposición de las estructuras universitarias en el régimen democrático vigente desde 1985 fue un proceso muy lento y los centros privados, particularmente CIEDUR, mantuvieron un papel importante para el desarrollo de la disciplina hasta entrada la década del noventa. Desde entonces puede sostenerse que la Historia Económica en Uruguay está prácticamente limitada a lo que se desarrolla en cuatro diferentes ámbitos de la Universidad de la República.

En la Facultad de Humanidades y Ciencias de la Educación la Historia Económica se cultiva de manera poco sistemática, y en ella no se realizan esfuerzos docentes específicos. La preocupación institucional ha estado centrada en el desarrollo de una Historia Total, aunque las áreas más dinámicas han sido las dirigidas por J. P. Barrán en torno al estudio de mentalidades, profesiones y sensibilidad. También se desarrollaron diversos proyectos de fuerte contenido histórico-económico, como estudios sobre empresarios, el sector energético, las elites coloniales, etc.

En la Facultad de Ciencias Económicas han existido dos nucleamientos vinculados a la disciplina. Por un lado se encuentran las cátedras de Historia Económica Nacional y Universal, dirigidas por B. Nahum. Estas cátedras, además de dictar los cursos correspondientes con una orientación más histórica que económica, han desarrollado una línea de investigación propia. En realidad, se ha tratado de una línea de trabajo más orientada a la ampliación de la base documental para la investigación que a la inves- 
tigación propiamente dicha. El trabajo ha consistido en la ubicación, traducción y publicación en forma de libros de informes diplomáticos británicos, franceses y belgas. Igualmente se han publicado volúmenes con información referente a la deuda externa y a episodios de importante repercusión diplomática, como la llamada «Reclamación Meillet». Se trata indudablemente de información valiosa que, dada la tecnología actualmente disponible y la utilidad que la información pudiera representar para la investigación, hubiese sido más útil presentarla de manera magnética que bajo la forma de libro.

En el Instituto de Economía de la mencionada facultad, a partir de la vuelta a la democracia, Julio Millot ha trabajado, con la colaboración de Magdalena Bertino, en un proyecto para redactar una historia económica del Uruguay en tres volúmenes. Después de la publicación del segundo, y habiéndose retirado Julio Millot de la actividad académica, se realizan actualmente esfuerzos por culminar ese trabajo.

Un núcleo dinámico de desarrollo de la disciplina ha estado en la creación, a comienzos de los años noventa, del Programa de Historia Económica y Social de la recientemente creada Facultad de Ciencias Sociales. Este programa logró reunir a investigadores provenientes de la historia y que habían permanecido trabajando en Uruguay en centros privados, como Raúl Jacob; a investigadores que retornaban a la Universidad después de haber vivido en el exterior, como el caso de Julio Millot, e investigadores más jóvenes con formación de doctorado en Historia Económica en el exterior, como es el caso del autor. Este programa nació con la vocación de consolidar una orientación de la disciplina que combinara los tres elementos mencionados en la introducción: el manejo de la teoría económica y social como instrumento indispensable para la investigación; el desarrollo de la crítica de las fuentes y el manejo sistemático de métodos cuantitativos, y la preocupación por los aspectos históricos e institucionales, por integrar la erudición histórica como elemento determinante de la interpretación histórica. Este programa puso entre sus principales objetivos la formación de nuevas generaciones de investigadores de acuerdo a estos lineamientos, y a partir del año 1994 incursionó en el dictado de cursos de posgrado, habiéndose iniciado una Maestría en Historia Económica en 1998.

Otro componente importante del mapa institucional uruguayo es la Asociación Uruguaya de Historia Económica (AUDHE), creada en 1992. Esta asociación nuclea al conjunto de investigadores activos en la disciplina y se ha constituido en una fértil herramienta para superar barreras ins- 
titucionales en lo interno y para el relacionamiento con la comunidad internacional.

Puede entonces sostenerse que, desde el punto de vista institucional, la Historia Económica ha experimentado un proceso de consolidación y especialización como fruto del desarrollo de la disciplina en periodos anteriores y canalizando lo realizado dentro de los centros privados hasta entrados los años noventa.

\section{Las líneas teórico-metodológicas y temáticas de la investigación}

\section{El contexto internacional}

No parece ser muy necesario abundar en el hecho de que, a nivel internacional, el desarrollo reciente del mundo que abarca el amplio espectro desde la Historia a la Economía se ha caracterizado por un divorcio entre esas dos grandes ramas de las ciencias sociales. ¿Qué andaba mal entre ellas? ¿Quién tuvo la culpa?

Desde el punto de vista de los historiadores la culpa es de los economistas. Ellos han sido fieles a aquello de que la ciencia no se propone tareas que no puede realizar. Las crecientes demandas de cientificidad, de poder de demostración matemática y empírica, ha llevado a una simplificación de las preguntas, de los enunciados y de las metodologías de investigación. El propio Solow, cuyo modelo de crecimiento ha sido sistemáticamente criticado con sus propias armas, resumía sagazmente esta tendencia ya hace unos cuantos años:

«My impression is that the best and brightest in the profession (economists) proceed as if economics were the physics of society. There is a single universally valid model of the world. It only needs to be applied. You could drop a modern economist from a time machine -a helicopter maybe, like the one that drops the money - at any time, in any place, along with his or her personal computer: he or she could set up in business without ever bothering to ask what time and which place. In a little while, the up-to-date economist will have maximized a familiar-looking present-value integral, made a few familiar log-linear approximates and run the obligatory familiar regression...» ${ }^{13}$.

${ }^{13}$ Solow (1985), p. 330. 
Hasta recientemente para la ciencia económica dominante algunos detalles quedaron por el camino: el factor tiempo, el cambio tecnológico, las «instituciones», entendiendo por esto las diferentes formas de armonización y confrontación de intereses sociales. A los historiadores, a su vez, las exigencias formales de los economistas los enfrentaba a demandas crecientes de cientificidad que aquéllos difícilmente podían satisfacer.

Para los economistas, los historiadores no sabían hacer Historia Económica: no usaban bien la teoría económica y cuando armaban información cuantitativa no la manejaban adecuadamente en términos económicos, ni la sometían adecuadamente a tests de constrastación de hipótesis. Los historiadores, entretanto, quedaron apuntando en otra dirección, manteniendo en alto la bandera de que la realidad es compleja, de que hay que abordarla de múltiples maneras. Desencantados con las macro-teorías, que mirando y desentrañando el pasado pretendían encontrar las claves para gobernar el desarrollo futuro, la investigación histórica comenzó a recorrer diferentes derroteros. En primer lugar, un alejamiento de los temas económicos. Éstos ya no explicaban todo: la conciencia, la cultura, la sensibilidad se transformaban en componentes mucho más difíciles de desentrañar y de guiar que lo que esperaban las profecías de cambio o los supuestos de los economistas. Se transformaban entonces en un objeto específico de análisis y las pretensiones del mismo eran mucho menos ambiciosas: de la mano de cierta de-teorización avanzan elementos de tipo más descriptivo y perceptivo. Por otra parte, las instituciones recuperan vigor en el análisis y la Historia política se remoza, desvinculándose definitivamente de las historias pragmáticas para profesionalizarse de la mano de la consolidación de la ciencia política como disciplina académica. Un elemento negativo acompaña esta positiva profesionalización de esta rama antiguamente tan desacreditada de la Historia: la vocación por la defensa de la especificidad de lo político, que contribuye, en cierta medida, a profundizar el divorcio entre la Economía y las ciencias sociales en general, además del ya mentado con la Historia. Un divorcio similar puede encontrase entre la sociología y la Historia Social.

Muchas veces los historiadores se han encontrado en una situación similar a los economistas, víctimas de ciertas opciones teórico-metodológicas (más metodológicas que teóricas). Estos últimos, seducidos por la elegancia formal y la demostración matemática, subyugados por los modelos y la informática, pierden de vista las grandes preguntas y las cuestiones relevantes, pierden de vista la importancia y pertinencia de los enunciados 
generales, prefieren, al decir de Crafts, estar precisamente equivocados antes que vagamente acertados ${ }^{14}$. Los historiadores, por su parte, ante las dificultades impuestas por la pérdida de poder explicativo de las grandes teorías y las diversas dificultades y exigencias metodológicas, muchas veces se han quedado meticulosamente reconstruyendo hechos, desempolvando información, aunque no siempre sabiendo qué buscar, qué preguntas formular, qué es lo que se quiere averiguar y qué importancia puede revestir en los debates de las ciencias sociales. El «surcido» de prolijas fichas domina la escena más de lo necesario, cuando no se ha estado repitiendo incansablemente las mismas convicciones de una y mil formas. Como en todos los divorcios, diría el terapeuta de esta pareja, los dos tienen su cuota de responsabilidad.

\section{La experiencia uruguaya}

Este divorcio llegó, sin duda, a las costas uruguayas. Como se anticipa del estudio institucional, este divorcio no llevó a la desaparición de la disciplina, sino a un estrechamiento relativo del número de cultores en relación especialmente a los años sesenta, al tiempo que a un proceso de especialización y maduración.

Desde el punto de vista de los economistas, encontramos principalmente un gran desinterés por los temas históricos y de largo plazo. Los temas de investigación, las herramientas de análisis, las preguntas planteadas privilegian nítidamente los enfoques de corto plazo. Muy pocos han sido los aportes de los economistas en esta dirección. No han dejado, sin embargo, de aparecer diversas contribuciones a la Historia Económica. Señalaremos algunas de ellas:

- El Banco Central del Uruguay financió un estudio del PBI uruguayo anterior a $1930{ }^{15}$. Este trabajo puso más énfasis en la sofisticación metodológica que en el trabajo con las fuentes históricas. Realizó peligrosas extrapolaciones de coeficientes técnicos de periodos posteriores y desestimó el uso de series más adecuadas proporcionadas por historiadores económicos, como es el caso de las series de comercio exterior producidas por Finch. Como resultado, esa contribución, si bien llenó un vacío informativo, no fue convincente.

is Crafts (1999), p. 5.

15 Arocena Olivera y Graziani (1987) y Arocena Olivera (1992). 
- Otra línea de trabajo ha estado compuesta de diversas interpretaciones de la relación entre crecimiento económico y marco institucional. La línea articuladora de estos trabajos ha sido la de demostrar lineales hipótesis acerca del papel benéfico de la liberalización. La gran mayoría de esta poca cantidad de trabajos ha mostrado muy poco interés por desentrañar los procesos históricos y por elaborar indicadores cuantitativos serios. Ha predominado una fuerte ideologización y parece ser ésta la corriente continuadora de las viejas corrientes de historiografía pragmática, es decir, aquella que pretende realizar una legitimación histórica de puntos de vista adoptados a priori, lo que habla de una seria debilidad de la vocación científica.

- Con algunos puntos de contacto con los aportes anteriores, pero de calidad sensiblemente superior, han aparecido unos pocos trabajos concentrados en el tratamiento de aspectos institucionales determinantes de los procesos de acumulación. Estos trabajos concluyen, paradójicamente, en que la explicación del desempeño económico radica en las características del sistema político ${ }^{16}$. Se desplaza así la problemática del desarrollo a una esfera no económica, al tiempo que parecería que la ciencia económica convencional no tiene grandes cosas para decir en relación a las determinantes de desempeños diversos.

Desde el campo de los historiadores hemos señalado que ha existido una pérdida de centralidad de los aspectos económicos. De todas formas, diversos proyectos de investigación se han mantenido en un campo muy cercano al de la Historia Económica, aunque sin ambiciones fuertes en el plano teórico-metodológico. A título de ejemplo, pueden señalarse los siguientes:

- Los trabajos de Alcides Beretta $(1993,1995,1996)$ se han concentrado en el seguimiento de trayectorias de vida de empresarios. Su valioso material se presenta con una impronta narrativa importante y se orienta más hacia una Historia Social.

- El de aspectos diplomáticos e institucionales relativos al desarrollo del sector energético ${ }^{17}$, que son de especial interés para los historiadores económicos.

Dentro de los trabajos claramente ubicados en el plano de la Historia Económica encontramos las siguientes principales contribuciones:

16 Ver, por ejemplo, Rama (1991).

17 Labraga, et al. (1991). 
- Los ya mencionados esfuerzos de recuperación documental de B. Nahum (1991-1997, 1993, 1998).

- Los trabajos de Raúl Jacob $(1988,1991)$ están más bien apegados a la tradición histórica. Su copiosa producción, recientemente nucleada en torno a las temáticas de la formación de grupos económicos y del poder económico, tiene a nuestro entender productos de especial destaque: su reconsideración del modelo del primer batllismo, en el que la política de construcción urbana y de infraestructura turística es jerarquizada, y su estudio del papel de los estudios jurídicos en el relacionamiento entre grupos económicos y poder político. Sin duda, su obra aborda invariablemente temas relevantes y su contribución a lo que McCloskey definiría como creación de más y mejores hechos económicos, tiene muy escasos parangones en la historiografía nacional. Dada la escasa preocupación de Jacob por explicitar y formalizar sus enfoques, éstos aparecen como excesivamente empiristas. Sin embargo, entre líneas pueden leerse rasgos de posturas teóricas más próximas al institucionalismo y a la escuela histórica alemana, cuya explicitación contribuiría a jeraquizar aún más los importantes aportes del autor.

- Entre los intentos por desarrollar la Historia Económica en la dirección de una ciencia social que mantiene ambiciones de generalización teórica, vocación de superación metodológica - tanto en el manejo de técnicas cuantitativas como cualitativas - e igualmente firmemente arraigada en la idea de que las ciencias sociales son necesariamente ciencias históricas, pueden encontrase los trabajos de Millot y Bertino $(1990,1996)$ sobre la historia económica del Uruguay desde la colonia hasta 1911. Estos trabajos presentan una gran cantidad de información y puede que hayan pecado por pretender abarcar demasiados tópicos. Su polémica con la hasta el momento incuestionada interpretación de Barrán y Nahum sobre el desarrollo ganadero de fines del siglo xxx, en especial el tema de la racionalidad de los estancieros, es de los puntos fuertes del trabajo. Sin embargo, la falta de actualización teórica en temas relevantes, junto a un manejo no del todo prolijo de la abundante base empírica, terminan dejando demasiado espacio para las valoraciones ideológicas.

- No soy el más indicado para evaluar mi propia producción. Los intentos por articular manejo teórico, metodología cuantitativa y contexto histórico son de difícil puesta en práctica y difícil es encontrar un equilibrio adecuado. Sin duda, mi esfuerzo ha sido mayor en los dos primeros aspectos, por entender que ésos eran los principales déficits en la historiografía 
nacional. Mi tesis de doctorado es mi trabajo individual más importante ${ }^{18}$. Mis aportes más recientes han priorizado los esfuerzos de reconstrucción de la base estadística $y$ han buscado absorber diversos desarrollos teóricos recientes para centrarse en el estudio comparativo del desempeño de largo plazo de la economia uruguaya y regional, aspecto que considero igualmente deficitario en la bistoriografía nacional ${ }^{19}$.

- Los esfuerzos del Programa de Historia Económica y Social por formar una nueva camada de investigadores en la disciplina van poco a poco dando resultados. De la primer experiencia de posgrados surgieron valiosos trabajos con ambición de renovación teórico-metodológica que auguran la progresiva consolidación de una relativamente amplia comunidad de investigadores tomando el desafío de desarrollar la disciplina en estrecha relación con su evolución internacional ${ }^{20}$.

\section{Situación y apuntes hacia una agenda}

Las referidas afirmaciones de McCloskey y Crafts nos pueden ayudar a sintetizar una evaluación del estado actual de la Historia Económica en el Uruguay. Ambos coinciden en que tanto la Historia Económica como la Economía contribuyen a crear más y mejores evidencias de hechos económicos. McCloskey habla luego de mejor teoría económica, mejor política económica y mejores economistas, mientras Cratfs habla de mejores hipótesis, mejor interpretación de la información y mejores historiadores.

Sin duda que la histórico-económica uruguaya ha producido una gran cantidad de hechos económicos a lo largo de su recorrido y que la calidad de los mismos se ha ido incrementando. Sin duda que los hechos económicos y su calidad tienen un fuerte contenido histórico, en el sentido que se evalúan en relación a criterios contemporáneos de lo que es mucho y de lo que es bueno. Frecuentemente se ha visto cómo ciertos conocimientos se devalúan y cómo los cambios de enfoque generan obsolescencia. Ello habla no sólo de la inexistencia de acumulación lineal; no es claro que siempre sobreviva lo mejor. La capacidad de seguir produciendo hechos económicos y mejorarlos, sin embargo, no es ajena a la capacidad de desarrollar y actualizar los cuerpos teóricos que construyen los hechos.

${ }^{18}$ Bértola (1990) (traducción al español 1992).

${ }^{19}$ Ver, por ejemplo, Bértola (1998), Bértola y Porcile (1998) y Bértola, Calicchio, Camou, Porcile (1999).

${ }^{20}$ Quisiéramos destacar los de Camou (1996), Calicchio (1996) y Moraes (1996). 
Si bien todos los componentes de ambas tipologías son indispensables para el desarrollo de las disciplinas en cuestión, existe cierta escala jerárquica a favor de los últimos elementos: mejores economistas e historiadores, mejor teoría económica e interpretación histórica, mejores hipótesis y política económica. Al avanzar en las caracterizaciones es donde la Historia Económica en Uruguay empieza a mostrar sus debilidades.

Si un programa de investigación y desarrollo de recursos humanos ha de aspirar a las metas mayores, debemos aceptar que la Historia Económica en Uruguay aún está poco desarrollada. Está produciendo una aún modesta cantidad de hechos económicos; está mejorando la calidad de los mismos en base a un esfuerzo de actualización teórica y empírica. Con respecto a la Economía, la Historia Económica uruguaya no ha contribuido de manera sustantiva, en su desarrollo reciente, ni a proveerla de mejores teorías, ni a formar mejores economistas, ni a mejorar las opciones de política económica. Obviamente, los economistas son ellos mismos responsables de ello, pero la oferta nacional en materia de Historia Económica no ha sido aún potente para hacerlos abrir los ojos. Con respecto a cómo los historiadores económicos han manejado la Economía para ser mejores historiadores, tener mejores hipótesis y realizar mejores interpretaciones, los avances son aún modestos, aunque parece ser más lo sembrado en esta dirección que en la opuesta. De aquí tal vez pueden surgir las líneas futuras de desarrollo y cierto optimismo. Veamos.

En otro trabajo (Bértola, 1999) he sostenido que el divorcio entre Economía e Historia Económica puede revertirse si quienes se dedican a la Historia Económica toman el desafío de aprovechar los desarrollos recientes que se están produciendo en la ciencia económica en la dirección de abordar decididamente los temas del cambio tecnológico y de la construcción institucional. Ambas direcciones de desarrollo de la Economía, presentes en diversas corrientes del pensamiento económico, llevan indefectiblemente a la historización y socialización de la teoría económica. Por otra parte, el desarrollo de las metodologías cuantitativas viene permitiendo un manejo más adecuado de procesos históricos complejos.

El desafío para los historiadores económicos parece ser el de combinar y anudar su conciencia histórica más desartollada, su conocimiento más amplio de los procesos históricos, con un fluido y audaz manejo de la teoría económica más sensible y consciente del cambio histórico y de sus connotaciones socio-institucionales, para lograr estilizar potentes y relevantes hechos y dinámicas históricas y contribuir así a desarrollar una teoría social e histórica del cambio económico. Puede que avances sístemáticos 
en esa dirección terminen por dar resultados que convenzan a los economistas de la utilidad de la Historia Económica y, a su vez, a los historiadores, de que hay teoría económica capaz de contribuir a un conocimiento histórico relevante.

En esta dirección las semillas están sembradas, y de perseverarse con continuidad y decisión en este camino, de profundizarse en las políticas de formación de jóvenes investigadores, de promoverse el intercambio regional e internacional de docentes y estudiantes, de mantenerse el objetivo de estar al día con el desarrollo internacional de la disciplina, la Historia Económica puede llegar, a pesar de la escasez de recursos, a tener un importante impacto en el medio académico uruguayo, en la formación de historiadores y economistas, en la conciencia histórica y en las propuestas de política. Sin duda que el éxito dependerá de poder articular estos modestos esfuerzos con lo que realicen los grandes vecinos. También en esa dirección hay avances, testimoniado, por ejemplo, por el carácter regional que recientemente han adoptado los congresos de Historia Económica realizados en los tres países.

\section{BIBLIOGRAFÍA}

Academia Nacional de Economia (ed.) (1984): Contribución a la Historia Económica del Uruguay, Montevideo.

ANICHINI, J. J.; CAUMONT, J., y SJAASTAD, L. (1977): La politica comercial y la protección en el Uruguay, BCU, Montevideo.

Arocena Olivera, E., y Graziani, C. (1987): El Ciclo Económico en el Uruguay entre 1866 y 1930 (Banco Central del Uruguay), Montevideo.

- (1992): Un enfoque cuantitativo de la economía uruguaya entre 1860 y 1935, Séptimas Jornadas Anuales de Economía, BCU, Montevideo.

Arrow, K. (1985): «Economic History: A necessary but not sufficient condition for an economist», American Economic Review, v. 75, 2/85, pp. 320-323.

Astori, D. (1979): La Evolución Tecnológica de la Ganadería Uruguaya 1930-79, Montevideo.

Barrán, J. P., y Nahum, B. (1967-1978): Historia Rural del Uruguay Moderno, I-VII, Montevideo.

- (1979-1985): Batlle, los Estancieras y el Imperio Británico, I-VII, Montevideo.

BerETTA, A. (1993): Pablo Varzi: un temprano espiritu de empresa, Montevideo.

- (1996): El Imperio de la Voluntad, Montevideo.

Beretta, A., y Garcia Etcheverry, A. (1995): Los burgueses inmigrantes, Montevideo.

BÉrTOLA, L. (1990): The Manufacturing Industry of Uruguay, 1913-1961: a sectoral approach to growth, fluctuations and crisis (Publications of the Department of Economic History, University of Göteborg, 61; Institute of Latin American 
Studies of Stockholm University, Monograph Nr 20). Gotemburgo-Estocolmo (traducción al español, 1992), La Industria Manufacturera Uruguaya 1913-1961. Un enfoque sectorial de su crecimiento, fluctuaciones y crisis, Montevideo.

- (1998): El PBI uruguayo 1870-1936 y otras estimaciones, Montevideo.

- (1999): Historia económica y economía: reflexiones sobre objeto y método, ponencia presentada al III Congresso Brasileiro de História Econômica, Curitiba.

BérTOLA, L., y Porcile, G. (1998): Argentina, Brazil, Uruguay and the World Economy: an approach to different convergence and divergence regimes (DT 42, Unidad Multidisciplinaria, Facultad de Ciencias Sociales), Montevideo.

Bértola, L.; Calicchio, L.; Camou, M., y Porcile, G. (1999): Southern Cone Real Wages Compared: a Purchasing Power Parity Approach to Convergence and Divergence Trends, 1870-1996 (DT 44, Unidad Multidisciplinaria, Facultad de Ciencias Sociales), Montevideo.

Calicchio, L. (1996): Salarios y Costo de Vida en el Rio de la Plata (1900-1930). (DT 33, Unidad Multidisciplinaria, Facultad de Ciencias Sociales), Montevideo.

CAmOU, M. M., y MORAES, M. I. (1998): Desarrollo reciente y perspectivas actuales de la bistoria económica en el Uruguay, ponencia presentada a las XVI Jornadas de Historia Económica Argentina, Quilmes.

Camou, M. M. (1996): Salarios y Costo de Vida en el Río de la Plata, 1880-1907 (DT 28, Unidad Multidisciplinaria, Facultad de Ciencias Sociales).

Crafts, N. F. R. (1999): «Quantitative Economic History» (Working Papers in Economic History 48/99), London School of Economics and Political Science, January.

De la Torre, N.; Rodríguez, J. C., y Sala de Tourón, L. (1971): Artigas: Tierra y Revolución, Montevideo.

De la Torre, N.; Sala de Tourón, L., y Rodriguez, J. C. (1972): Después de Artigas (1820-1836), Montevideo.

FAROPPA, L. (1965): El Desarrollo Económico del Uruguay. Tentativa de Explicación, Montevideo.

Finch, H. (1980): Historia Económica del Uruguay Contemporáneo, Montevideo [edición en inglés Finch, M. H. J. (1981): A Political Economy of Uruguay Since 1870, Londres y Basingstoke].

JACOB, R. (1981): Depresión Ganadera y Desarrollo Fabril, Montevideo.

- (1983): El Uruguay de Terra 1931-1938, Montevideo.

- (1988): Modelo Batllista: ¿Variación sobre un Viejo Tema?, Montevideo.

- (1991): 1915-1945. Las otras dinastias, Montevideo.

LABRAGA, A., et. al. (1991): Energía y política en el Uruguay del siglo XX, Montevideo.

MCClOSkeY, D. (1976): «Does the Past Have Useful Economics», Journal of Economic Literature, vol. XIV, núm. 2, pp. 434-461.

Millot, J., y BerTino, M. (1990): Historia económica del Uruguay, tomo I, Montevideo.

- (1996): Historia económica del Uruguay, tomo II, Montevideo.

Millot, J.; Silva, C., y Silva, L. (1973): El Desarrollo Industrial del Uruguay. De la Crisis de 1929 a la Post-guerra de la Segunda Guerra Mundial (Departamento de Publicaciones de la Universidad de la República), Montevideo. 
Moraes, M. I. (1996): La Política Económica para el Complejo Productor de Cames Vacunas en Argentina y Uruguay (1930-1959), (DT 31, Unidad Multidisciplinaria, Facultad de Ciencias Sociales), Montevideo.

NAHUM, B. (1991-1997): Informes diplomáticos de los representantes del Reino Unido en el Uruguay, tomos I-VII, Montevideo.

- (1993): La «Reclamación Meillet» (Serie Documental, I), Montevideo.

- (1998): Informes diplomáticos de los representantes de Bélgica en el Uruguay, Montevideo.

NORTH, D. (1994): Some fundamental puzzles in economic bistory/development, p. 1. Tomado de su website.

Rama, M. (1991): El país de los vivos. Un enfoque económico (Sextas Jornadas Anuales de Economía, BCU).

Sala de Tourón, L.; De la Torre, N.; Rodríguez, J. C. (1978): Artigas y su revolución agraria. 1811-1820, Montevideo.

Sala de Tourón, L.; De la Torre, N., y Rodriguez, J. C. (1967): Estructura económica y social de la colonia, Montevideo.

Sala de Tourón, L.; Rodriguez, J., y De la TORRE, N. (1967): Evolución económica de la Banda Oriental, Montevideo.

Sen, A. (1988): «The Concept of Development» en Chenery, H., y SRINIVASAN, T. N. (eds.), Handbook of Development Economics, vol. I.

Solow, R. (1985): «Economic History and Economics», American Economic Review, v. 75, 2/85, pp. 325.30.

Uruguay, CIDE (Comisión de Inversiones y Desarrollo Económico) (1963): Estudio Económico del Uruguay: Evolución y Perspectivas, I-II, Montevideo.

Uruguay, MGA (Ministerio de Ganadería y Agricultura) (1967): OPYPA-CIDE, 5-Estudio Económico y Social de la Agricultura en el Uruguay, I-II. Montevideo.

Uruguay, Universidad de la República, Instituto de Economía (1968): El Proceso Económico del Uruguay, Montevideo. 